\title{
Application of CHROMagar candida, A New Differential Isolation Medium for Presumptive Identification of candida Species from Oral Cavity of Immunocompromised Patients
}

\author{
Jyoti. D. Magare ${ }^{1}$, Dr. R. S. Awasthi ${ }^{2}$ \\ ${ }^{1}$ C.S.M.S.S. Dental College, Aurangabad (M.S). India \\ ${ }^{2}$ Shivaji College, Renapur, Latur (M.S).India
}

\begin{abstract}
CHROMagar Candida (CA) is a new differential culture medium that allows selective isolation of yeasts and simultaneously identifies colonies of Candida albicans, Candida. tropicalis, , Candida parapsilosis and Candida krusei. In comparison with conventional medium i.e. sabrauads dextrose agar (SDA), we evaluated 50 isolates directly on CHROMagar and SDA from oral samples. Over $95 \%$ of clinical isolates of Candida albicans, Candida tropicalis, Candida parapsilosis and Candida krusei were correctly identified on the basis of colony morphology and pigmentation on CHROMagar as compared to SDA. CHROMagar is extremely useful in making a rapid presumptive identification of common yeast species. This capability plus the ability to detect mixed cultures of Candida spp. promises to improve and streamline the work flow in the mycology and clinical microbiology laboratory.
\end{abstract}

Keywords: CHROMagar Candida (CA), sabrauads dextrose agar (SDA), conventional medium, rapid presumptive identification

\section{Introduction}

During the past two decades, there has been a significant increase in the prevalence of fungal infections caused by Candida species. The yeast candida being the main cause of candidiasis is commonly isolated pathogen from immunocompromised patients. The trend in the resistance acquired by some species of candida leads to the importance of identification to the species level in immunocompromised patients. Lesions of thrush are seen in children and adults of all ages whenever the number of candida in the oral cavity increases significantly (1). In routine diagnostic laboratories Sabouraud dextrose agar (SDA) is widely used for the isolation of all yeast species from clinical specimens (2). CHROMagar Candida (CA), a yeast differential and selective medium, allows the recognition of mixed yeast cultures in clinical specimens. The medium permits the presumptive identification of $C$. albicans from other Candida spp. Yeast populations are differentiated by colonial morphology and colours which are generated by a chromophore in the agar (3) $C$. albicans strains produce $P$ $\mathrm{N}$-acetyl galactosaminidase, which interacts with the chromophore (chromogenic hexosaminidase substrate) incorporated into the agar, and with incubation for $48 \mathrm{~h}$ produces green colonies, characteristic of all $C$. albicans isolates (4).

The objective of this study was to examine the use of CHROMagar as the primary or follow-up medium for the culture and identification of yeasts in a routine laboratory.

\section{Materials and Methods}

Sample Collection: Specimens from oral lesions were collected by passing sterile cotton swab several times across the surface, including oral thrush. Immediately after sampling, each swab was replaced in sterile tube and transported within 30 minutes of sampling from the place of collection of the laboratory.

Culture Media: CHROMagar and Sabouraud dextrose agar (SDA) containing Chloramphenicol were prepared as per manufacturer's instruction. All the collected samples were inoculated directly on SDA and CHROMagar. The inoculated plates were kept for incubation for $24 \mathrm{hrs}$.

Identification Methods: All yeast isolates observed on CHROMagar were identified by colony morphology and pigmentation according to the manufacturer's instructions and as described by Odds and Bernarets (3). Isolates on SDA shows creamy white colored colonies shows presence of Candida .Macroscopic (creamy white colonies) and microscopic (yeast cell pseudohyphae and blastospores) examination of the growth verified by diagnosis of candidiasis. The isolated colonies are tested for identification of species (5). The isolated colonies from SDA are subculture on corn meal agar plate and incubated at $25^{\circ} \mathrm{C}$ for 48-72 hrs. Candida albicans species was identified microscopically by production of chlamydospores (6). The germ tube test to identify candida albicans was done according to the method of Cheesbrough. About $0.5 \mathrm{ml}$ of human blood serum was poured into small tube and each isolate were inoculated into tube using sterile wire loop. Incubation was in a water bath for 2-4 hrs. A drop of serum yeast culture was transferred onto a glass slide using a Pasteur pipette and covered with a cover slip. Examination for germ tubes or blastospores was done under the $40 \mathrm{X}$ objective. The presence of sprouting yeast cells was a positive identification for C. albicans (6).Germ tube negative and chlamydospores negative species are further identified by biochemical and assimilation test for different non candida albicans species. 


\section{International Journal of Science and Research (IJSR) \\ ISSN (Online): 2319-7064 \\ Index Copernicus Value (2013): 6.14 | Impact Factor (2014): 5.611}

\section{Results}

A total number of 50 isolates were analyzed in this study These organisms were fresh isolates, all of which were cultured from specimens.

\section{Sample Collection}

The isolates were from oral thrush of patients suffering from oral candidiasis.

\section{Identification}

Culture on SDA shows pure colonies which are creamy white colour with yeasty odour. All Candida albicans shows germ tube formation and also they form chlamydospores on corn meal agar. Candida species utilized glucose, galactose, sucrose and maltose. All the non albicans candida utilized starch; Candida albicans utilized raffinose in addition to the four sugars in common. The development of the colony color was noted on CHROMagar Candida, and we observed that the medium better differentiated the color of colonies after 48 hours of incubation, while after 72 hours of incubation a distinct deep color of the colonies was observed. The color of colonies on CHROMagar Candida was similar as given by the manufacturer, i.e. green colonies of Candida albicans. blue colonies of Candida tropicalis, pink colored colonies of Candida krusei, and Candida parapsilosis produced pinkish white colonies.(Fig .no.1)

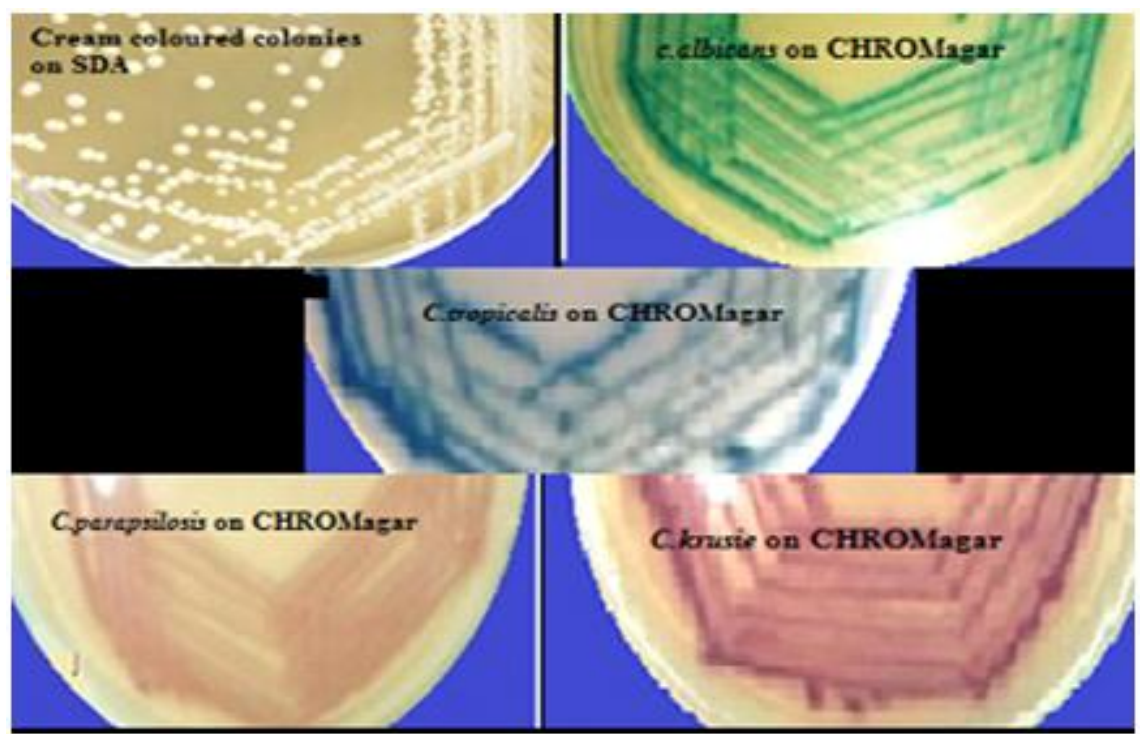

Figure 1: Creamed coloured colonies on SDA. 2. C.albicans on CHROMagar 3. C.tropicalis on CHROMagar 4. C. parapsilosis on CHROMagar 5. C.krusie on CHROMagar

\section{Discussion}

CHROMagar Candida is an easy and reliable method for the presumptive identifications of most commonly isolated Candida species (7).CHROMagar-Candida was originally developed for the selective isolation and presumptive identification of some clinically important yeast species such as $C$. albicans, C. tropicalis, $C$. krusei and C. glabrata on the basis of differences in color and surface of colonies (8).

Of the 50 clinical specimens, 47 candida species were isolated from oral cavity. Candida albicans was found to be the predominant with $86 \%$ followed by Candida krusei, $2 \%$ Candida tropicalis 4\% Candida parapsilosis 2\%. Overall 47 Candida species were isolated on SDA and CHROMagar. CHROMagar appeared somewhat more sensitive than SDA in detecting Candida albicans, Candida krusei, Candida parapsilosis and Candida tropicalis.(As shown in table no.1)
Table 1: The identification of Candida species based on standard routine Sabourauds dextrose agar (SDA) and HiCrome Candida agar

\begin{tabular}{|c|c|c|c|}
\hline Species & $\begin{array}{c}\text { Conventional } \\
\text { method (SDA) } \\
\text { Number } \\
\mathrm{N}=50\end{array}$ & $\begin{array}{c}\text { Morphology } \\
\text { Colour }\end{array}$ & $\begin{array}{c}\text { Sensitivity } \\
\text { Percentage }\end{array}$ \\
\hline C. albicans & 43 & Light green & $100 \%$ \\
\hline C. krusei & 01 & Pink & $100 \%$ \\
\hline C. tropicalis & 02 & Blue & $100 \%$ \\
\hline C.parapsilosis & 01 & Light pink & $100 \%$ \\
\hline
\end{tabular}

Our studies confirm the previous investigations regarding the high accuracy of CHROMagar. We found presumptive identification of $100 \%$ of Candida albicans isolates, Candida parapsilosis Candida tropicalis and Candida krusei isolates. None of the remaining species were misidentified as one of these four species. The results of this study confirm those of Odds and Bernarets (3) regarding the accuracy of CHROMagar in providing a presumptive identification of $C$. albicans, C. tropicalis, and C.krusei. Beighton et al. (4) have reported that CHROMagar-Candida is a very useful medium and its use will facilitate the study of yeasts associated with dental diseases. It is reported by Odds et al. (3) that using of CHROMagar-Candida was an extremely successful differential detector of yeasts that provides no required germ tube confirmation for $C$.

\section{Volume 4 Issue 11, November 2015}




\section{International Journal of Science and Research (IJSR) \\ ISSN (Online): 2319-7064}

Index Copernicus Value (2013): 6.14 | Impact Factor (2014): 5.611

albicans. Likely to this report, Pfaller et al. (9) have reported that stool or rectal swabs cultures obtained from surgical and neonatal intensive care units do not perform identifications beyond a germ tube test in case of using CHROMagarCandida.

Yucesoy and Marol (10) reported that the sensitivity and specificity rates for CHROMagar-Candida were $99.4100 \%$ for $C$. albicans, respectively. They reported the sensitivity of CHROMagar-Candida to identify C. tropicalis, C. glabrata, and $C$. krusei ranged between 90.0 and $100 \%$ while the specifity values were found to be 95.4 and 100 for $C$. tropicalis and C. krusei, respectively.

Our results are also in agreement with these prior studies although the isolates were recovered from oral swabs. In agreement with the earlier studies, sensitivity and specificity were determined $100 \%$ in our study which was carried on with a larger spectrum of samples of C. albicans, C. krusei, $C$. parapsilosis and $C$. tropicalis. In addition to these studies, Fotedar and al-Hedaithy (11) have reported that CHROMAgar-Candida is not only a simple, reliable and cost effective method for the identification of chlamydospore-negative atypical $C$. albicans, but can also be used to differentiate various groups of chlamydospores negative $C$. albicans.

In conclusion, CHROMagar provides a simple and inexpensive method for the identification of yeasts. This should allow laboratories to introduce identification of mucosal isolates, now increasingly required and recommended with minimal impact on workflows and laboratory budgets.

\section{References}

[1] Magare JD, and Awasthi RS.,(2014). Evaluating the Prevalence of Candida Species in the Oral Cavity of Immunocompromised Patients. IJSR. 3 (3). 180-183.

[2] Odds FC. Sabouraud ('s) agar. J Med Vet Mycol 1991; 29:355-359.

[3] Odds FC, Bernarets R.,(1994) CHROMagar Candida, a new differential isolation medium for presumptive identification of clinically important Candida species. $J$ CIin Microbiol 32.1923-1929.

[4] Beighton D, Ludford R, Clark DT et al.(1995) Use of CHROMagar Candida medium for isolation of yeasts from dental samples. J Clin Microbiol 33: 3025-3027.

[5] Jawetz, Melnick, Adelberg: Text book of Medical Microbiology Publisher Mc Graw Hill Medical:24 ${ }^{\text {th }}$ Edition: Chapter 45:632-43.

[6] Shaheen M A. and Taha M. (2006), Species Identification of candida Isolates obtained from oral lesions of Hospitalized and Non Hospitalized patients with oral candidiasis, Egyptian Dermatology Online Journal: 2(14).1-14.

[7] Year, H., Poulain, D., Lefebvre, A.,(2004) "Polymicrobial candidemia revealed by peripheral blood smear and chromogenic medium" J. Clin. Pathol., 57, 196-198,

[8] Tornai-Lehoczki, J., Peter, G., Dlauchy, D.,(2003) "CHROMAgar-Candida medium as a practical tool for the differentiation and presumptive identification of yeast species isolated from salads" Int. J. Food Microbiol., 86, 189-200,

[9] Pfaller, M.A., Houston, A., Coffmann, S.,( 1996). "Application of CHROMagar-Candida for rapid Screening of Clinical specimens for Candida albicans, Candida tropicalis, Candida krusei, and Candida (torulopsis) glabrata "J.Clin. Microbiol., 34, 58-61,

[10] Yucesoy, M., Marol, S.,(2003) "Performance of CHROMagar and BIGGY agar for identification of yeast species" Ann. Clin. Microbiol. Antimicrob., 2(8), $1-7$.

[11]Fotedar, R., al-Hedaithy, S.S.,(2003) "Identification of chlamydospores-negative Candida albicans using CHROM Agar-Candida medium” Mycoses, 46, 96-103.

\section{Author Profile}

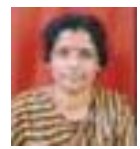

Ms Jyoti. D. Magare received her M. Sc degree in Microbiology in the year 1996. In the year 1997 joined as Assistant Professor in C. S. M. S. S. Dental College Aurangabad (M S). India. Participated and presented the poster in International conference held at Pune in 2013.published paper in thematic journal of microbiology in 2013. On her credit one Indian patent is registered and published in patent journal.

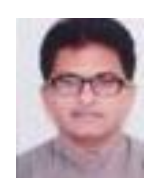

Dr. R. S. Awasthi is Principal at Shivaji college Renapur Dist Latur. He is research guide for Ph.D. and M. Phil. student at Swami Ramanand Teerth Marathwada University Nanded, Dr Babasaheb Ambedkar Marathwada University Aurangabad, NIIMS University, YCMOU University Nashik. He has Written Two books and worked as editor for three books of reputed publisher. On his credit two Indian patent is registered and published in patent journal.. He successfully guided 8 Ph.D. students and five M. Phil. students. He completed two research project sponsored by UGC New Delhi. He visited Bhutan Shrilanka and Nepal and delivered talk.He is a member of American Board of Advisors, West Calina (US).He is a non govt member of committee for granting permission for biotechnology course in Maharashtra. 